Acta Crystallographica Section A

Foundations of Crystallography

ISSN 0108-7673

Received 23 January 2003

Accepted 8 April 2003

C 2003 International Union of Crystallography Printed in Great Britain - all rights reserved

\section{Determination of all misorientations of tetragonal lattices with low multiplicity; connection with Mallard's rule of twinning}

\author{
Hans Grimmer \\ Laboratory for Neutron Scattering, ETHZ and PSI, CH-5232 Villigen PSI, Switzerland. \\ Correspondence e-mail: hans.grimmer@psi.ch
}

Two congruent lattices are considered, which are misoriented in such a way that they have a fraction $1 / \Sigma$ of symmetry translations in common. Whereas for cubic lattices body or face centring does not affect the 'multiplicity' or 'twin index' $\Sigma$, this is not generally true for tetragonal lattices. Consider a fixed misorientation and let $\Sigma_{P}$ and $\Sigma_{I}$ be the multiplicities for $t P$ and $t I$ lattices with the same axial ratio c/a. Grimmer [Mater. Sci. Forum (1993), 126-128, 269-272] has given an explicit formula for $\Sigma_{P}$ (depending on the misorientation and the axial ratio) and showed that $\Sigma_{I}=\Sigma_{P} / 2, \Sigma_{P}$ or $2 \Sigma_{P}$. Here stronger results on the occurrence of the three possibilities are presented. Lists of all axial ratios $c / a$ of $t P$ and $t I$ lattices admitting misorientations with $\Sigma \leq 5$ are given. For each of these misorientations, the twin mirror planes and their normals are listed, so that a synopsis of all possible twin laws of tetragonal crystals by reticular merohedry with $\Sigma \leq 5$ is obtained. It is shown that the two twin laws observed in $\beta$-Sn can be described by reticular pseudomerohedry with $\Sigma_{I}=2$ and obliquity $\delta=2.6134^{\circ}$.

\section{Introduction}

Mallard (1885) distinguished different types of twins, which were characterized by Friedel (1926) in terms of the twin index $\Sigma$ and the obliquity $\delta$. Friedel distinguished four cases according to whether $\Sigma=1$ or $\Sigma>1$ and whether $\delta=0$ or $\delta>0$, and stated that the observed twins have $\Sigma \leq 5$ and $\delta<6^{\circ}$ (with $\delta<4^{\circ}$ in most cases). The statement that twins satisfy such limits on $\Sigma$ and $\delta$ is usually referred to as Mallard's law or Mallard's rule. Recently, Le Page (2002) developed a convenient program, which computes the twin laws satisfying Mallard's rule for a specified crystal lattice. The present paper considers twinning of tetragonal crystals by reticular merohedry (i.e. the case $\Sigma>1, \delta=0$ ). It lists all possible twin laws with $\Sigma \leq 5$ and applies the results to twinning of tin by reticular pseudomerohedry $(\Sigma>1, \delta>0)$.

If the misorientation of the two lattices of the twin can be described by a rotation about the tetragonal axis then $\Sigma$ will be the same for tetragonal primitive $(t P)$ and body-centred $(t I)$ lattices and will not depend on the axial ratio $c / a$. These are the common misorientations, for which $\delta=0$. If $c^{2} / a^{2}$ is a rational number, then there exist additional specific misorientations. Grimmer (1993) developed methods that make it possible to determine all $t P$ lattices that allow specific misorientations with $\Sigma$ less than a chosen limit. They will be summarized in $\$ 2$ and applied in $\$ 3$ to list all $t P$ lattices that allow specific misorientations with $\Sigma \leq 5$. The effect of body centring on the twin index is investigated in $\S 4$. Similarly to $\S 3$ for $t P$ lattices, we list in $\S 5$ for each $c / a$ value of a $t I$ lattice the possible twin indices $\Sigma \leq 5$ and the corresponding axes of $180^{\circ}$ rotations and twin mirror planes in a standard spherical triangle, i.e. the possible twin laws by reticular merohedry. Similar results for $h P$ and $h R$ lattices have been obtained by Grimmer (1989). In $\S 6$, the results for $t I$ lattices are applied to tin and compared with experimental findings and with the results of Le Page (2002). The results of the paper are discussed in $\$ 7$.

\section{Coincidence misorientations of tetragonal lattices}

The coincidence site lattice (CSL) model (see e.g. the review by Fischmeister, 1985) of grain boundaries in polycrystalline materials claims that neighbouring grains often have a large proportion of symmetry translations in common. This proportion can be characterized as follows. Let $V$ be the volume of a smallest (i.e. primitive) cell spanned by the symmetry translations of one of the grains and $V_{C}$ the volume of the smallest cell spanned by the symmetry translations that the two grains have in common. Then the ratio $\Sigma=V_{C} / V$ is called the multiplicity (of the CSL). We speak of a coincidence site lattice if $V_{C}$ is finite, in which case $\Sigma$ is a finite integer $\geq 1$. Also the lattice generated by the translations that leave either of the two grains invariant is of physical interest. Bollmann (1967) called it the complete pattern-shift lattice or 'displacement shift complete lattice' (DSC lattice or DSCL); its vectors are the geometrically possible Burgers vectors of 
Table 1

The common misorientations with $\Sigma \leq 50$.

Each misorientation is given by its representative quadruple $(m, U, V, W)$ and the corresponding minimum rotation angle $\Theta$. Also the axes $[u v w]$ of equivalent $180^{\circ}$ rotations in the standard spherical triangle are listed; the axis $[u v w]$ and the plane $(h k l)$ perpendicular to it, called twin mirror plane, have the same indices.

\begin{tabular}{|c|c|c|c|c|c|c|}
\hline \multirow[b]{2}{*}{$\Sigma$} & \multirow[b]{2}{*}{$\omega$} & \multicolumn{2}{|c|}{ Representative } & \multicolumn{3}{|c|}{ Twin mirror planes } \\
\hline & & $\Theta^{\circ}$ & $(m, U, V, W)$ & $\begin{array}{l}{[u v w]} \\
(h k l)\end{array}$ & $\begin{array}{l}{[u v w]} \\
(h k l)\end{array}$ & $\begin{array}{l}{[u v w]} \\
(h k l)\end{array}$ \\
\hline 1 & 1 & 0 & 1000 & 100 & 110 & 001 \\
\hline 5 & 2 & 36.87 & 3001 & 310 & 210 & \\
\hline 13 & 2 & 22.62 & 5001 & 510 & 320 & \\
\hline 17 & 2 & 28.07 & 4001 & 410 & 530 & \\
\hline 25 & 2 & 16.26 & 7001 & 710 & 430 & \\
\hline 29 & 2 & 43.60 & $\begin{array}{llll}5 & 0 & 0 & 2\end{array}$ & 520 & 730 & \\
\hline 37 & 2 & 18.92 & 60001 & 610 & 750 & \\
\hline 41 & 2 & 12.68 & 9001 & 910 & 540 & \\
\hline
\end{tabular}

perfect grain-boundary dislocations. The volume $V_{D}$ of the smallest cell of the DSCL is $V_{D}=V / \Sigma$.

The misorientation of two congruent tetragonal lattices can be described by a rotation relating conventional bases of the two lattices. Some rotations lead to CSLs in every tetragonal lattice. These are the common rotations, for which $\Sigma$ does not depend on the axial ratio $c / a$. If $c^{2} / a^{2}$ is a rational number, then there exist additional specific rotations. ${ }^{1}$ Common and specific rotations can be characterized by quadruples of integers $(m, U, V, W)$ without a common divisor, where $[U V W]$ is the axis and

$$
\Theta=2 \arctan \left\{\frac{1}{m}\left[\frac{a^{2}\left(U^{2}+V^{2}\right)+c^{2} W^{2}}{c^{2}}\right]^{1 / 2}\right\}
$$

the angle of the rotation. The angle $\Theta$ has the same sign as $m$. The rotation with angle $|\Theta|$ around $[U V W]$ is anticlockwise (right-handed) for $m>0$ and clockwise for $m<0$. The quadruple $(-m, U, V, W)$ describes the inverse rotation to $(m, U, V, W)$, but the rotations for $\pm(m, U, V, W)$ are identical. Owing to the rotational symmetry 422 of the tetragonal lattice, which contains 8 elements, and the interchangeability of the two congruent lattices, there are in general $2 \times 8 \times 8=128$ rotations expressing the same misorientation; they will be called equivalent. For certain misorientations, several of these rotations are identical. The actual number of distinguishable equivalent rotations is always a multiple of 8 and a divisor of 128 , and will be written as $8 \omega, \omega=1,2,4,8$ or 16 (Grimmer, 1980). The number of different equivalent quadruples is $16 \omega$ because $\pm(m, U, V, W)$ describe the same rotation. A quadruple $(m, U, V, W)$ describes a common rotation if either $U=$ $V=0$ or $m=W=0$. In the first case, we have a rotation with axis $\| \mathbf{c}$, in the second a $180^{\circ}$ rotation with axis $\perp \mathbf{c}$.

Among equivalent rotations with the same rotation angle, there is at least one with axis in the standard spherical triangle: $U \geq V \geq 0, W \geq 0$. Such a rotation with minimum angle, called the reduced rotation, and the corresponding quadruple

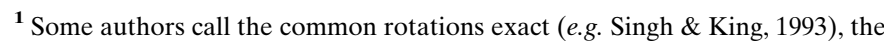
specific rotations approximate or constrained.
}

Table 2

The values $\mu, v$ for which the lower bound (3) is $\leq 5$ and the actual minimum of $\Sigma_{P}$.

\begin{tabular}{|c|c|c|c|}
\hline \multirow[t]{2}{*}{$\mu$} & \multicolumn{3}{|l|}{$v$} \\
\hline & & & \\
\hline$v$ & $\mu$ & Lower bound for $\Sigma_{P}$ & Minimum of $\Sigma_{P}$ \\
\hline 1 & 1 & 1 & 1 \\
\hline 2 & 1 & 2 & 2 \\
\hline 3 & 1 & 1.73 & 2 \\
\hline 4 & 1 & 2 & 2 \\
\hline 5 & 1 & 2.24 & 3 \\
\hline 6 & 1 & 3.46 & 4 \\
\hline 7 & 1 & 2.65 & 4 \\
\hline 8 & 1 & 2.83 & 3 \\
\hline 9 & 1 & 3 & 3 \\
\hline 10 & 1 & 4.47 & 6 \\
\hline 11 & 1 & 3.32 & 6 \\
\hline 12 & 1 & 3.46 & 4 \\
\hline 13 & 1 & 3.61 & 7 \\
\hline 15 & 1 & 3.87 & 4 \\
\hline 16 & 1 & 4 & 4 \\
\hline 17 & 1 & 4.12 & 9 \\
\hline 19 & 1 & 4.36 & 10 \\
\hline 20 & 1 & 4.47 & 6 \\
\hline 21 & 1 & 4.58 & 5 \\
\hline 23 & 1 & 4.80 & 12 \\
\hline 24 & 1 & 4.90 & 5 \\
\hline 25 & 1 & 5 & 5 \\
\hline 3 & 2 & 3.46 & 4 \\
\hline 5 & 2 & 4.47 & 6 \\
\hline 4 & 3 & 3.46 & 4 \\
\hline 5 & 3 & 3.87 & 4 \\
\hline 7 & 3 & 4.58 & 5 \\
\hline 8 & 3 & 4.90 & 5 \\
\hline 5 & 4 & 4.47 & 6 \\
\hline
\end{tabular}

( $m, U, V, W$ ) with non-negative components is typically chosen to represent the misorientation (Singh \& King, 1993).

Most sets of equivalent rotations for CSLs with a low value of $\Sigma$ contain also $180^{\circ}$ rotations. They are of particular interest because one can show (for lattices of any Bravais class) that (a) the axis of the $180^{\circ}$ rotation is a twofold symmetry axis of the CSL defined by this rotation and $(b)$ the multiplicity can be computed in a simple way.

For a given value of $c / a$ and a given misorientation, the multiplicity will generally depend on the centring type of the lattice. It will be called $\Sigma_{P}$ in the case of $t P$ lattices and $\Sigma_{I}$ for $t I$ lattices. For common misorientations we have $\Sigma_{P}=\Sigma_{I}=\Sigma$. Those with $\Sigma \leq 50$ are listed in Table 1 .

\section{Coincidence misorientations of $\boldsymbol{t P}$ lattices with $\Sigma \leq \mathbf{5}$}

For primitive tetragonal $(t P)$ lattices, a generally valid formula for computing $\Sigma_{P}$ has been given by Grimmer (1993). Here we shall give an alternative formulation, distinguishing nine cases. Consider two congruent $t P$ lattices with $c^{2} / a^{2}=\mu / \nu$, where $\mu$ and $v$ are integers with greatest common divisor equal to 1 , g.c.d. $(\mu, v)=1$, and write $\mu=2^{M} \mu_{0}, v=2^{N} v_{0}$, where $\mu_{0}$ and $v_{0}$ are odd. If the misorientation of the two lattices is given by the quadruple ( $m, U, V, W)$ with g.c.d. $(m, U, V, W)=1$, then the multiplicity of the CSL will be 


$$
\Sigma_{P}=\frac{\mu\left(m^{2}+W^{2}\right)+v\left(U^{2}+V^{2}\right)}{\alpha \text { g.c.d. }\left(\mu_{0}, U, V\right) \text { g.c.d. }\left(v_{0}, m, W\right)},
$$

where $\alpha$ is a power of 2 given by

$$
\begin{array}{lll}
\alpha=\text { g.c.d. }\left(4, m^{2}+U^{2}+V^{2}+W^{2}\right) & \text { if } M=N=0 \\
\alpha=2 \text { g.c.d. }\left(2^{M}, 2 U, 2 V, U+V\right) & \text { if } M>0, & U, V, m+W \text { even } \\
\alpha=\text { g.c.d. }\left(2^{M}, 2 U, 2 V\right) & \text { if } M>0, & U, V \text { even } n, m+W \text { odd } \\
\alpha=2 & \text { if } M>0, & U, V \text { odd } \\
\alpha=1 & \text { if } M>0, & U+V \text { odd } \\
\alpha=2 \text { g.c.d. }\left(2^{N}, 2 m, 2 W, m+W\right) & \text { if } N>0, & m, W, U+V \text { even } \\
\alpha=\text { g.c.d. }\left(2^{N}, 2 m, 2 W\right) & \text { if } N>0, & m, W \text { even, } U+V \text { odd } \\
\alpha=2 & \text { if } N>0, & m, W \text { odd } \\
\alpha=1 & \text { if } N>0, & m+W \text { odd }
\end{array}
$$

Grimmer (1993) derived the following lower bound for $\Sigma_{P}$ :

$$
\begin{array}{ll}
\Sigma_{P} \geq(\mu \nu)^{1 / 2} & \text { if } \mu \nu \text { is odd or a multiple of } 4, \\
& \text { otherwise } \Sigma_{P} \geq(2 \mu \nu)^{1 / 2} .
\end{array}
$$

This result tells us that only the pairs $\mu, v$ listed in Table 2 may give rise to $\Sigma_{P} \leq 5$. The table shows that for 19 of the 29 entries the actual minimum value of $\Sigma_{P}$ is the minimum integer satisfying the lower bound. The difference between our lower bound and the actual minimum increases if $\mu$ or $v$ is a large prime.

Using a computer program developed by Grimmer (1993), the misorientations leading to small values of $\Sigma_{P}$ have been calculated for the $2 \times 28+1$ pairs $\mu, v$ in the table. Exchanging $\mu$ and $\nu$ does not affect the minimum value of $\Sigma_{P}$, which is listed in Table 2. All specific misorientations of $t P$ lattices with $\Sigma_{P} \leq 5$ are given in Table 3.

Notice the twofold symmetry of Table 3: if $\mu$ and $v$ are interchanged, the same values of $\omega$ appear for the same $\Sigma_{P}$ values. The row with $\mu=v=1$ corresponds to a lattice that metrically coincides with the primitive cubic lattice $(c P)$. For this lattice, there exist several misorientations with $\Sigma_{P}=3$ and $\Sigma_{P}=5$ that are not equivalent with respect to the tetragonal holohedry (although they are equivalent with respect to the cubic holohedry).

\begin{tabular}{|c|c|c|c|c|c|c|c|c|}
\hline \multirow[b]{2}{*}{$\mu$} & \multirow[b]{2}{*}{$v$} & \multirow[b]{2}{*}{$c^{2} / a^{2}$} & \multirow[b]{2}{*}{$c / a$} & \multicolumn{5}{|c|}{$\omega$ for $\Sigma_{P}=$} \\
\hline & & & & 1 & 2 & 3 & 4 & 5 \\
\hline 1 & 25 & 0.04 & 0.2 & & & & & 2 \\
\hline 1 & 24 & 0.0417 & 0.2041 & & & & & 4 \\
\hline 1 & 21 & 0.0476 & 0.2182 & & & & & 4 \\
\hline 1 & 16 & 0.0625 & 0.25 & & & & 2 & 4 \\
\hline 1 & 15 & 0.0667 & 0.2582 & & & & 4 & \\
\hline 1 & 12 & 0.0833 & 0.2887 & & & & 4 & 4 \\
\hline 1 & 9 & 0.1111 & 0.3333 & & & 2 & & 4 \\
\hline 1 & 8 & 0.125 & 0.3536 & & & 4 & 2 & 4 \\
\hline 1 & 7 & 0.1429 & 0.3780 & & & & 4 & \\
\hline 1 & 6 & 0.1667 & 0.4082 & & & & 4 & 4 \\
\hline 1 & 5 & 0.2 & 0.4472 & & & 4 & & 8 \\
\hline 1 & 4 & 0.25 & 0.5 & & 2 & 4 & & 4 \\
\hline 1 & 3 & 0.3333 & 0.5774 & & 4 & & 8 & 4 \\
\hline 3 & 8 & 0.375 & 0.6124 & & & & & 4 \\
\hline 3 & 7 & 0.4286 & 0.6547 & & & & & 4 \\
\hline 1 & 2 & 0.5 & 0.7071 & & 2 & 4 & & 4 \\
\hline 3 & 5 & 0.6 & 0.7746 & & & & 4 & \\
\hline 2 & 3 & 0.6667 & 0.8165 & & & & 4 & 4 \\
\hline 3 & 4 & 0.75 & 0.8660 & & & & 4 & 4 \\
\hline 1 & 1 & 1 & 1 & 2 & & 8,4 & & $4,4,8$ \\
\hline 4 & 3 & 1.3333 & 1.1547 & & & & 4 & 4 \\
\hline 3 & 2 & 1.5 & 1.2247 & & & & 4 & 4 \\
\hline 5 & 3 & 1.6667 & 1.2910 & & & & 4 & \\
\hline 2 & 1 & 2 & 1.4142 & & 2 & 4 & & 4 \\
\hline 7 & 3 & 2.3333 & 1.5275 & & & & & 4 \\
\hline 8 & 3 & 2.6667 & 1.6330 & & & & & 4 \\
\hline 3 & 1 & 3 & 1.7321 & & 4 & & 8 & 4 \\
\hline 4 & 1 & 4 & 2 & & 2 & 4 & & 4 \\
\hline 5 & 1 & 5 & 2.2361 & & & 4 & & 8 \\
\hline 6 & 1 & 6 & 2.4495 & & & & 4 & 4 \\
\hline 7 & 1 & 7 & 2.6458 & & & & 4 & \\
\hline 8 & 1 & 8 & 2.8284 & & & 4 & 2 & 4 \\
\hline 9 & 1 & 9 & 3 & & & 2 & & 4 \\
\hline 12 & 1 & 12 & 3.4641 & & & & 4 & 4 \\
\hline 15 & 1 & 15 & 3.8730 & & & & 4 & \\
\hline 16 & 1 & 16 & 4 & & & & 2 & 4 \\
\hline 21 & 1 & 21 & 4.5826 & & & & & 4 \\
\hline 24 & 1 & 24 & 4.8990 & & & & & 4 \\
\hline 25 & 1 & 25 & 5 & & & & & 2 \\
\hline
\end{tabular}

Tables 4 and 5 give for each misorientation listed in Table 3 the quadruple representing the reduced rotation and the corresponding axes of $180^{\circ}$ rotations and twin mirror planes in the standard spherical triangle $U \geq V \geq 0, W \geq 0$. The misorientations are arranged according to increasing values of $\Sigma_{P}$ and, for a given value of $\Sigma_{P}$, according to increasing values of $c / a$. Instead of the angle $\Theta$ that corresponds to the representative quadruple $(m, U, V, W)$ according to equation (1), we listed $i=2 \Sigma_{P} \cos \Theta$. This takes less space and makes it possible to determine $\Theta$ with unrestricted precision because $i$ is an integer.

The representative rotations for the entries with $\omega=2$ or 4 in Tables 4 and 5 have axis [100] or [110]. (These are axes of twofold symmetry rotations of the tetragonal lattice.) If $\omega=2$ then $i=0$, i.e. $\Theta=90^{\circ}$; there is only one twin mirror plane with axis in the standard spherical triangle (SST); CSL and DSCL have at least tetragonal symmetry. If $\omega=4$ then $i \neq 0$, i.e. $\Theta \neq$ $90^{\circ}$; there are two twin mirror planes with axis in the SST; CSL and DSCL have at least orthorhombic symmetry. The repre-
Table 3

The axial ratios $c / a$ of primitive tetragonal lattices for which there exist specific misorientations leading to $\Sigma_{P} \leq 5$.

For each $c / a$, the corresponding values of $\Sigma_{P}$ are given with their $\omega$ values.

sentative rotation for the entries with $\omega=8$ has axis different from [100] and [110]; CSL and DSCL have at least monoclinic symmetry. These results remain true for $t I$ lattices ( $c f$. Tables 9 and 10).

Tables 4 and 5 allow us to read off all axes of $180^{\circ}$ rotations and all twin mirror planes of $t P$ twins by reticular merohedry, with $\Sigma_{P} \geq 5$, as well as the corresponding values of $c / a$. If the experimental value of the axial ratio slightly deviates from one of these values, then the corresponding laws describe twinning by reticular pseudomerohedry $(\delta>0)$.

\section{The effect of body centring on the coincidence site lattice}

Consider two congruent primitive tetragonal lattices, 1 and 2, with common translation vectors forming a three-dimensional CSL. In the following, we discuss how the multiplicity of the CSL changes if we pass from $t P$ to $t I$ lattices. This can be done 
Table 4

Specific misorientations with multiplicity $\Sigma_{P} \leq 4$ for the $t P$ lattices.

\begin{tabular}{|c|c|c|c|c|c|c|c|c|c|c|}
\hline \multirow[b]{2}{*}{$\Sigma_{P}$} & \multirow[b]{2}{*}{$\mu$} & \multirow[b]{2}{*}{$v$} & \multirow[b]{2}{*}{$\omega$} & \multirow[b]{2}{*}{$c / a$} & \multicolumn{2}{|c|}{ Representative } & \multicolumn{2}{|c|}{ Twin mirror plane 1} & \multicolumn{2}{|c|}{ Twin mirror plane 2} \\
\hline & & & & & $(m, U, V, W)$ & $i$ & $(h k l)$ & {$\left[\begin{array}{lll}u & v & w\end{array}\right]$} & $(h k l)$ & {$\left[\begin{array}{lll}u & v & w\end{array}\right]$} \\
\hline 1 & 1 & 1 & 2 & 1. & 1100 & 0 & 101 & 101 & & \\
\hline \multirow[t]{6}{*}{2} & 1 & 4 & 2 & 0.5 & 2100 & 0 & 201 & 102 & & \\
\hline & 1 & 3 & 4 & 0.5774 & 3100 & 2 & 101 & 103 & 301 & 101 \\
\hline & 1 & 2 & 2 & 0,7071 & 2110 & 0 & 111 & 112 & & \\
\hline & 2 & 1 & 2 & 1.4142 & 1110 & 0 & 112 & 111 & & \\
\hline & 3 & 1 & 4 & 1.7321 & 1100 & 2 & 103 & 101 & 101 & 301 \\
\hline & 4 & 1 & 2 & 2. & 1200 & 0 & 102 & 201 & & \\
\hline \multirow[t]{12}{*}{3} & 1 & 9 & 2 & 0.3333 & 3100 & 0 & 301 & 103 & & \\
\hline & 1 & 8 & 4 & 0.3536 & 4100 & 2 & 201 & 104 & 401 & 102 \\
\hline & 1 & 5 & 4 & 0.4472 & 5100 & 4 & 101 & 105 & 501 & 101 \\
\hline & 1 & 4 & 4 & 0.5 & 4110 & 2 & 111 & 114 & 221 & 112 \\
\hline & 1 & 2 & 4 & 0.7071 & 2100 & 2 & 101 & 102 & 201 & 101 \\
\hline & 1 & 1 & 8 & 1. & 3111 & 3 & 211 & 211 & & \\
\hline & 1 & 1 & 4 & 1. & 2110 & 2 & 112 & 112 & 111 & 111 \\
\hline & 2 & 1 & 4 & 1.4142 & 1100 & 2 & 102 & 101 & 101 & 201 \\
\hline & 4 & 1 & 4 & 2. & 1110 & 2 & 114 & 111 & 112 & 221 \\
\hline & 5 & 1 & 4 & 2.2361 & 1100 & 4 & 105 & 101 & 101 & 501 \\
\hline & 8 & 1 & 4 & 2.8284 & 1200 & 2 & 104 & 201 & 102 & 401 \\
\hline & 9 & 1 & 2 & 3. & 1300 & 0 & 103 & 301 & & \\
\hline \multirow[t]{20}{*}{4} & 1 & 16 & 2 & 0.25 & 4100 & 0 & 401 & 104 & & \\
\hline & 1 & 15 & 4 & 0.2582 & 5100 & 2 & 301 & 105 & 501 & 103 \\
\hline & 1 & 12 & 4 & 0.2887 & 6100 & 4 & 201 & 106 & 601 & 102 \\
\hline & 1 & 8 & 2 & 0.3536 & 4110 & 0 & 221 & 114 & & \\
\hline & 1 & 7 & 4 & 0.3780 & 7100 & 6 & 101 & 107 & 701 & 101 \\
\hline & 1 & 6 & 4 & 0.4082 & 6110 & 4 & 111 & 116 & 331 & 112 \\
\hline & 1 & 3 & 8 & 0.5774 & 3111 & 1 & 211 & 213 & & \\
\hline & 3 & 5 & 4 & 0.7746 & 5300 & 2 & 101 & 305 & 503 & 101 \\
\hline & 2 & 3 & 4 & 0.8165 & 3110 & 4 & 112 & 113 & 332 & 111 \\
\hline & 3 & 4 & 4 & 0.8660 & 2100 & 4 & 203 & 102 & 201 & 302 \\
\hline & 4 & 3 & 4 & 1.1547 & 3200 & 4 & 102 & 203 & 302 & 201 \\
\hline & 3 & 2 & 4 & 1.2247 & 2110 & 4 & 113 & 112 & 111 & 332 \\
\hline & 5 & 3 & 4 & 1.2910 & 1100 & 2 & 305 & 101 & 101 & 503 \\
\hline & 3 & 1 & 8 & 1.7321 & 3331 & 1 & 213 & 211 & & \\
\hline & 6 & 1 & 4 & 2.4495 & 1110 & 4 & 116 & 111 & 112 & 331 \\
\hline & 7 & 1 & 4 & 2.6458 & 1100 & 6 & 107 & 101 & 101 & 701 \\
\hline & 8 & 1 & 2 & 2.8284 & 1220 & 0 & 114 & 221 & & \\
\hline & 12 & 1 & 4 & 3.4641 & 1200 & 4 & 106 & 201 & 102 & 601 \\
\hline & 15 & 1 & 4 & 3.8730 & 1300 & 2 & 105 & 301 & 103 & 501 \\
\hline & 16 & 1 & 2 & 4. & 1400 & 0 & 104 & 401 & & \\
\hline
\end{tabular}

Table 5

Specific misorientations with multiplicity $\Sigma_{P}=5$ for the $t P$ lattices.

\begin{tabular}{|c|c|c|c|c|c|c|c|c|c|}
\hline \multirow[b]{2}{*}{$\mu$} & \multirow[b]{2}{*}{$v$} & \multirow[b]{2}{*}{$\omega$} & \multirow[b]{2}{*}{$c / a$} & \multicolumn{2}{|c|}{ Representative } & \multicolumn{2}{|c|}{ Twin mirror plane 1} & \multicolumn{2}{|c|}{ Twin mirror plane 2} \\
\hline & & & & $(m, U, V, W)$ & $\bar{i}$ & $\overline{(h k l)}$ & {$[u v w]$} & $\overline{(h k l)}$ & {$[u v w$} \\
\hline 1 & 25 & 2 & 0.2 & 5100 & 0 & 501 & 105 & & \\
\hline 1 & 24 & 4 & 0.2041 & 6100 & 2 & 401 & 106 & 601 & 104 \\
\hline 1 & 21 & 4 & 0.2182 & 7100 & 4 & 301 & 107 & 701 & 103 \\
\hline 1 & 16 & 4 & 0.25 & 8100 & 6 & 201 & 108 & 801 & 102 \\
\hline 1 & 12 & 4 & 0.2887 & 6110 & 2 & 221 & 116 & 331 & 114 \\
\hline 1 & 9 & 4 & 0.3333 & 9100 & 8 & 101 & 109 & 901 & 101 \\
\hline 1 & 8 & 4 & 0.3536 & 8110 & 6 & 111 & 118 & 441 & 112 \\
\hline 1 & 6 & 4 & 0.4082 & 3100 & 2 & 201 & 103 & 301 & 102 \\
\hline 1 & 5 & 8 & 0.4472 & 5210 & 0 & 211 & 215 & & \\
\hline 1 & 4 & 4 & 0.5 & 4100 & 6 & 101 & 104 & 401 & 101 \\
\hline 1 & 3 & 4 & 0.5774 & 3110 & 2 & 111 & 113 & 332 & 112 \\
\hline 3 & 8 & 4 & 0.6124 & 2100 & 2 & 403 & 102 & 201 & 304 \\
\hline 3 & 7 & 4 & 0.6547 & 7300 & 4 & 101 & 307 & 703 & 101 \\
\hline 1 & 2 & 4 & 0.7071 & 4110 & 6 & 112 & 114 & 221 & 111 \\
\hline 2 & 3 & 4 & 0.8165 & 3200 & 2 & 101 & 203 & 302 & 101 \\
\hline 3 & 4 & 4 & 0.8660 & 2110 & 2 & 223 & 112 & 111 & 334 \\
\hline 1 & 1 & 4 & 1 & 3100 & 8 & 103 & 103 & 301 & 301 \\
\hline 1 & 1 & 4 & 1 & 2100 & 6 & 102 & 102 & 201 & 201 \\
\hline 1 & 1 & 8 & 1 & 3311 & -1 & & & & \\
\hline
\end{tabular}


Table 5 (continued)

\begin{tabular}{|c|c|c|c|c|c|c|c|c|c|}
\hline \multirow[b]{2}{*}{$\mu$} & \multirow[b]{2}{*}{$v$} & \multirow[b]{2}{*}{$\omega$} & \multirow[b]{2}{*}{$c / a$} & \multicolumn{2}{|l|}{ Representative } & \multicolumn{2}{|c|}{ Twin mirror plane 1} & \multicolumn{2}{|c|}{ Twin mirror plane 2} \\
\hline & & & & $(m, U, V, W)$ & $i$ & $(h k l)$ & {$[u v w]$} & $(h k l)$ & {$\left[\begin{array}{lll}u & v & w\end{array}\right]$} \\
\hline 4 & 3 & 4 & 1.1547 & 3220 & 2 & 112 & 223 & 334 & 111 \\
\hline 3 & 2 & 4 & 1.2247 & 1100 & 2 & 203 & 101 & 101 & 302 \\
\hline 2 & 1 & 4 & 1.4142 & 2110 & 6 & 114 & 112 & 111 & 221 \\
\hline 7 & 3 & 4 & 1.5275 & 1100 & 4 & 307 & 101 & 101 & 703 \\
\hline 8 & 3 & 4 & 1.6330 & 3400 & 2 & 102 & 403 & 304 & 201 \\
\hline 3 & 1 & 4 & 1.7321 & 1110 & 2 & 113 & 111 & 112 & 332 \\
\hline 4 & 1 & 4 & 2 & 1100 & 6 & 104 & 101 & 101 & 401 \\
\hline 5 & 1 & 8 & 2.2361 & 1210 & 0 & 215 & 211 & & \\
\hline 6 & 1 & 4 & 2.4495 & 1200 & 2 & 103 & 201 & 102 & 301 \\
\hline 8 & 1 & 4 & 2.8284 & 1110 & 6 & 118 & 111 & 112 & 441 \\
\hline 9 & 1 & 4 & 3 & 1100 & 8 & 109 & 101 & 101 & 901 \\
\hline 12 & 1 & 4 & 3.4641 & 1220 & 2 & 116 & 221 & 114 & 331 \\
\hline 16 & 1 & 4 & 4 & 1200 & 6 & 108 & 201 & 102 & 801 \\
\hline 21 & 1 & 4 & 4.5826 & 1300 & 4 & 107 & 301 & 103 & 701 \\
\hline 24 & 1 & 4 & 4.8990 & 1400 & 2 & 106 & 401 & 104 & 601 \\
\hline 25 & 1 & 2 & 5 & 1500 & 0 & 105 & 501 & & \\
\hline
\end{tabular}

more easily if the lattices are taken as point lattices with at least one point in common. The CSL then consists of all those points that are common to the two lattices. The body centring is carried out on lattice 1 first. A new net plane is inserted in this way between each pair of neighbouring net planes perpendicular to the fourfold axis of lattice 1 . The old coincidence points lie in the old net planes. If the centring produces additional coincidence sites, then these sites must lie in the new net planes; the distance between neighbouring net planes of the CSL decreases by a factor of 2 in this case. Analogous considerations hold for the net planes perpendicular to the fourfold axis of lattice 2 if the body centring is consecutively carried out on that lattice. Because the density of the lattice points is always the same for parallel net planes, one obtains the following result: $\Sigma_{I}=\Sigma_{P} / 2, \Sigma_{P}$ or $2 \Sigma_{P}$ according to whether both, one or none of the two centrings produces new net planes of the CSL. From this result and the lower bound (2) for $\Sigma_{P}$, it follows that

$$
\begin{array}{ll}
\Sigma_{I} \geq(\mu \nu)^{1 / 2} / 2 & \text { if } \mu \nu \text { is odd or a multiple of } 4, \\
& \text { otherwise } \Sigma_{I} \geq(\mu \nu / 2)^{1 / 2}
\end{array}
$$

(Grimmer, 1993).

Next I shall show how this lower bound can be made more stringent. If $c^{2} / a^{2}=\mu / v$, g.c.d. $(\mu, v)=1$, we choose the length units of our conventional tetragonal coordinate system such that $c^{2}=\mu$ and $a^{2}=v$. It follows that a vector $[u v w]$ has length square $L^{2}([u v w])=v\left(u^{2}+v^{2}\right)+\mu w^{2}$. The length squares of the vectors of the $t P$ lattice are therefore integers. In order to obtain a body-centred lattice, we generate new vectors $\frac{1}{2}[u v w]$, where $[u v w]$ is a vector of lattice 1 with $u, v, w$ all odd. Such a vector cannot coincide with a vector of the $t P$ lattice 2 unless $L^{2}\left(\frac{1}{2}[u v w]\right)=\frac{1}{4}\left\{v\left(u^{2}+v^{2}\right)+\mu w^{2}\right\}$ is an integer. Because the square of an odd number has the form $(2 k+1)^{2}=4 k(k+1)+1$ and at least one of the numbers $\mu, v$ is odd, this is possible only if $\mu$ is an even number that is not a multiple of 4 . Only in this case is it possible that $\Sigma_{I}=\Sigma_{P} / 2$.
Using the lower bound (2) on $\Sigma_{P}$ and the result obtained above, we find the following lower bound for the multiplicity of the CSL in $t I$ lattices:

$$
\begin{gathered}
\Sigma_{I} \geq(\mu \nu)^{1 / 2} \quad \text { if } \mu \nu \text { is odd or a multiple of } 4 \\
\text { otherwise } \Sigma_{I} \geq(\mu \nu / 2)^{1 / 2} \quad \text { if } \mu \text { is even, } \\
\Sigma_{I} \geq(2 \mu \nu)^{1 / 2} \quad \text { if } \nu \text { is even. }
\end{gathered}
$$

This result will be applied in the following section to determine the pairs $\mu, v$ that give rise to $\Sigma_{I} \leq 5$.

Next we shall discuss examples illustrating the three cases $\Sigma_{I}=\Sigma_{P} / 2, \Sigma_{P}$ or $2 \Sigma_{P}$. Fig. 1 shows from left to right the last three entries with $\Sigma_{P}=2$ in Table 4, giving the three lattices before and after the $180^{\circ}$ rotation about the axis $[u v w]$ indicated in column 'Twin mirror plane 1' of Table 4. This axis is plotted perpendicular to the plane of the drawing and passing through point $O$. Fig. 1(a) shows planes in which all lattice points belong to the CSL. Because $\Sigma_{P}=2$ in all three cases, the points of $t P$ in the neighbouring lattice plane do not belong to the CSL of $t P$. Fig. 1(b) shows a lattice plane of $t I$ next to the plane given in Fig. 1(a). This plane contains also points of $t P$ except in the case shown on the right, where the additional points of $t I$ lie halfway between lattice planes of $t P$. In summary, whereas for $t P$ all lattice points belong to the CSL in every second lattice plane parallel to the plane of the figure, for $t I$ all lattice points belong to the CSL in every lattice plane (left), every second lattice plane (middle), and every fourth lattice plane (right), i.e. $\Sigma_{I}=1,2$ and 4, respectively.

In the following, we shall show that $\Sigma_{P}$ is odd and $\Sigma_{I}=\Sigma_{P}$ if $\mu$ and $v$ are odd and differ by a multiple of 4 [i.e. if either $\mu \cong v \cong 1(\bmod 4)$ or $\mu \cong v \cong 3(\bmod 4)]$. This can be proved as follows: consider $f=\mu\left(m^{2}+W^{2}\right)+v\left(U^{2}+V^{2}\right)$ and $f^{\prime}=m^{2}+$ $W^{2}+U^{2}+V^{2}$, where $m, U, V, W$ are coprime integers. The number of factors 2 in $f$ (and $f^{\prime}$ ) is 0 if one or three of the four integers $m, U, V, W$ are odd, it is 1 if two are odd, and 2 if all four are odd. (Being coprime, the four numbers $m, U, V, W$ cannot all be even.) It follows then from (2) that $\Sigma_{P}$ is odd.

The vectors of the $t P$ lattice 1 can be classified into 8 types according to whether their components are even $(e)$ or odd 
Table 6

The squares of the lengths of vectors with even $(e)$ and odd $(d)$ components up to multiples of 4 for the cases $\mu \cong v \cong 1(\bmod 4)$ and $\mu \cong v \cong 3(\bmod 4)$.

\begin{tabular}{lll}
\hline Vector types & $\mu \cong v \cong 1$ & $\mu \cong v \cong 3$ \\
\hline$[e e e]$ & 0 & 0 \\
{$[d e e],[e d e],[e e d]$} & 1 & 3 \\
{$[e d d],[d e d],[d d e]$} & 2 & 2 \\
{$[d d d]$} & 3 & 1 \\
\hline
\end{tabular}

(d). If $\mathbf{v}$ is a vector of the CSL, then $2 \mathbf{v}$ is a vector of the CSL of type [eee]. The CSL vectors of this type form a subgroup $U$ of the group $G$ of all vectors of the CSL. The volume of a primitive cell is $\Sigma_{P}$ for the lattice $G$ and a multiple of 8 for the lattice $U$. We conclude that the number of cosets of $U$ in $G$ is (a multiple of) 8 if $\Sigma_{P}$ is odd, i.e. that the CSL must contain vectors of all 8 possible combinations of even and odd components. For the squared lengths of the vectors of the 8 types, we obtain the results given in Table 6 .

The vectors of the CSL of type $[d d d]$ in the coordinates of lattice 1 must be of the same type in lattice 2 because vectors of other types cannot have the same length according to Table 6. We conclude: if $[u v w]$ is a vector of type $[d d d]$ of the CSL of a primitive tetragonal lattice, then $\frac{1}{2}[u v w]$ is a vector of the CSL of the corresponding body-centred lattice. This completes the proof that $\Sigma_{I}=\Sigma_{P}$ if $\mu$ and $v$ are odd and differ by a

(a)

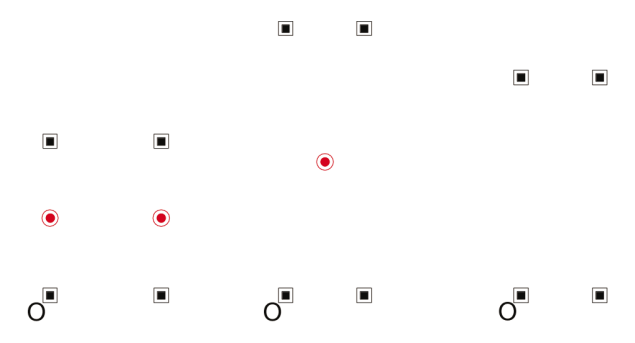

(b)
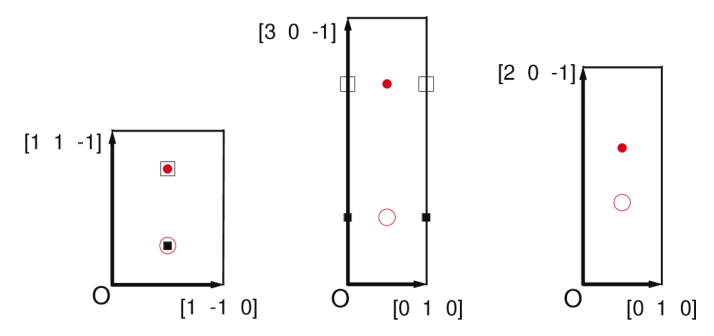

$$
c^{2} / a^{2}=2
$$

3

4

lattice planes

perpendicular to $\left[\begin{array}{lll}1 & 1 & 1\end{array}\right] \quad\left[\begin{array}{lll}1 & 0 & 1\end{array}\right]$

$\left[\begin{array}{lll}2 & 0 & 1\end{array}\right]$

\section{Figure 1}

Three twin misorientations for which $\Sigma_{P}=2$ and $\Sigma_{I}=1$ (left), $\Sigma_{I}=2$ (middle), $\Sigma_{I}=4$ (right). The squares mark the points of $t P$, squares and circles together the points of $t I$. The full symbols correspond to lattice points before, the open symbols after the $180^{\circ}$ rotation with axis through $O$ perpendicular to the plane of the drawing. (a) shows planes of $t I$, in which the points of the two lattices coincide; $(b)$ shows lattice planes next to $(a)$.

Table 7

The twin index $\Sigma_{I}$ for $t I$ lattices expressed in terms of $S=|h u+k v+l w|$ and of $\Sigma_{P}$.

\begin{tabular}{llll}
\hline$h+k+l$ & $u, v, w$ & $S$ & $\Sigma_{I}$ \\
\hline \multirow{2}{*}{ Odd } & & $S$ even & $S=2 \Sigma_{P}$ \\
\multirow{3}{*}{ Even } & $S$ odd & $S=\Sigma_{P}$ \\
& Not all odd & $S$ odd & $S=\Sigma_{P}$ \\
& & $S$ even & $S / 2=\Sigma_{P}$ \\
& All odd & $S / 2$ odd & $S / 2=\Sigma_{P}$ \\
& & $S / 2$ even & $S / 4=\Sigma_{P} / 2$ \\
\hline
\end{tabular}

multiple of 4 . For the case $\mu \cong v \cong 1(\bmod 4)$, this result was already stated (without proof) in Grimmer (1993).

The actual calculation of $\Sigma_{P}$ and $\Sigma_{I}$ is easy if the misorientation can be described by a $180^{\circ}$ rotation. The multiplicities $\Sigma_{P}$ and $\Sigma_{I}$ then coincide with the twin index introduced by Friedel (1926), which (for any lattice, not just tetragonal ones) is simply related to the axis $[u v w]$ of the $180^{\circ}$ rotation and the Miller indices $(h k l)$ of the plane perpendicular to $[u v w]$, as given by Friedel (1926) and reproduced in Table 3.1.9 of Donnay \& Donnay (1959). For tetragonal lattices with rational value of $c^{2} / a^{2}$, their results can be formulated as follows: put $c^{2} / a^{2}=\mu / \nu$, where $\mu$ and $v$ are positive integers satisfying g.c.d. $(\mu, v)=1$, and let the axis of the $180^{\circ}$ rotation be [uvw] with g.c.d. $(u, v, w)=1$. Then the Miller indices $(h k l)$ with g.c.d. $(h, k, l)=1$ of the plane perpendicular to $[u v w]$ are

$$
(h k l)=\frac{(v u v v \mu w)}{\text { g.c.d. }(\mu, u, v) \text { g.c.d. }(v, w)} .
$$

It follows that the quantity $S=|h u+k v+l w|$ considered by Donnay \& Donnay (1959) equals

$$
S=\frac{v\left(u^{2}+v^{2}\right)+\mu w^{2}}{\text { g.c.d. }(\mu, u, v) \text { g.c.d. }(v, w)} .
$$

For $t P$ lattices, Friedel (1926) obtained $\Sigma_{P}=S$ if $S$ is odd, $\Sigma_{P}=$ $S / 2$ if $S$ is even. It is not difficult to show that this result agrees with the special case $m=0$ of our formula (2). Friedel's results for $\Sigma_{I}$ are given in Table 7 .

Computer calculations suggest additional relations between $\Sigma_{I}$ and $\Sigma_{P}: \Sigma_{I} \neq 2 \Sigma_{P}$ if $\mu \cong 2(\bmod 4)$, and $\Sigma_{I}=\Sigma_{P}$ if $\mu$ and $v$ are odd. Whereas a general proof of these two relations has not yet been given, it can be shown that they are true if the misorientation can be expressed by a $180^{\circ}$ rotation. The proof makes use of equation (5) and Table 7.

\section{Coincidence misorientations of $\boldsymbol{t}$ l lattices with $\Sigma \leq \mathbf{5}$}

These misorientations were calculated starting out with equation (4) to determine possible pairs $\mu$, $\nu$. For these pairs $\mu, v$, the misorientations with low values of $\Sigma_{P}$ were calculated and the corresponding values of $\Sigma_{I}$ determined, making use of the fact that $\Sigma_{I}$ coincides with Friedel's twin index when the misorientation can be described by a $180^{\circ}$ rotation. For $\Sigma_{I} \leq 5$, this is the case with only two exceptions, which correspond to $t I$ lattices that metrically agree with the cubic body-centred $(c I)$ and face-centred $(c F)$ lattice, respectively. For cubic 
Table 8

The axial ratios $c / a$ of body-centred tetragonal lattices for which there exist specific misorientations leading to $\Sigma_{I} \leq 5$.

For each $c / a$, the corresponding values of $\Sigma_{I}$ are given with their $\omega$ values.

\begin{tabular}{|c|c|c|c|c|c|c|c|c|}
\hline \multirow[b]{2}{*}{$\mu$} & \multirow[b]{2}{*}{$v$} & \multirow[b]{2}{*}{$c^{2} / a^{2}$} & \multirow[b]{2}{*}{$c / a$} & \multicolumn{5}{|c|}{$\omega$ for $\Sigma_{I}=$} \\
\hline & & & & 1 & 2 & 3 & 4 & 5 \\
\hline 1 & 25 & 0.04 & 0.2 & & & & & 2 \\
\hline 1 & 21 & 0.0476 & 0.2182 & & & & & 4 \\
\hline 1 & 15 & 0.0667 & 0.2582 & & & & 4 & \\
\hline 2 & 25 & 0.08 & 0.2828 & & & & & 2 \\
\hline 2 & 21 & 0.0952 & 0.3086 & & & & & 4 \\
\hline 1 & 9 & 0.1111 & 0.3333 & & & 2 & & 4 \\
\hline 2 & 15 & 0.1333 & 0.3651 & & & & 4 & \\
\hline 1 & 7 & 0.1429 & 0.3780 & & & & 4 & \\
\hline 1 & 6 & 0.1667 & 0.4082 & & & & & 4 \\
\hline 1 & 5 & 0.2 & 0.4472 & & & 4 & & 8 \\
\hline 2 & 9 & 0.2222 & 0.4714 & & & 2 & & 4 \\
\hline 1 & 4 & 0.25 & 0.5 & & & & 2 & 4 \\
\hline 2 & 7 & 0.2857 & 0.5345 & & & & 4 & \\
\hline 1 & 3 & 0.3333 & 0.5774 & & 4 & & 8 & 4 \\
\hline 2 & 5 & 0.4 & 0.6325 & & & 4 & & 8 \\
\hline 3 & 7 & 0.4286 & 0.6547 & & & & & 4 \\
\hline 1 & 2 & 0.5 & 0.7071 & & & 4 & 2 & 4 \\
\hline 3 & 5 & 0.6 & 0.7746 & & & & 4 & \\
\hline 2 & 3 & 0.6667 & 0.8165 & & 4 & & 8 & 4 \\
\hline 6 & 7 & 0.8571 & 0.9258 & & & & & 4 \\
\hline 1 & 1 & 1 & 1 & 2 & & $8+4$ & & $4+4+8$ \\
\hline 6 & 5 & 1.2 & 1.0954 & & & & 4 & \\
\hline 4 & 3 & 1.3333 & 1.1547 & & & & & 4 \\
\hline 3 & 2 & 1.5 & 1.2247 & & & & & 4 \\
\hline 5 & 3 & 1.6667 & 1.2910 & & & & 4 & \\
\hline 2 & 1 & 2 & 1.4142 & 2 & & $4+8$ & & $8+4+4$ \\
\hline 7 & 3 & 2.3333 & 1.5275 & & & & & 4 \\
\hline 3 & 1 & 3 & 1.7321 & & 4 & & 8 & 4 \\
\hline 10 & 3 & 3.3333 & 1.8257 & & & & 4 & \\
\hline 4 & 1 & 4 & 2 & & & 4 & 2 & 4 \\
\hline 14 & 3 & 4.6667 & 2.1602 & & & & & 4 \\
\hline 5 & 1 & 5 & 2.2361 & & & 4 & & 8 \\
\hline 6 & 1 & 6 & 2.4495 & & 4 & & 8 & 4 \\
\hline 7 & 1 & 7 & 2.6458 & & & & 4 & \\
\hline 8 & 1 & 8 & 2.8284 & & & & 2 & 4 \\
\hline 9 & 1 & 9 & 3 & & & 2 & & 4 \\
\hline 10 & 1 & 10 & 3.1623 & & & 4 & & 8 \\
\hline 12 & 1 & 12 & 3.4641 & & & & & 4 \\
\hline 14 & 1 & 14 & 3.7417 & & & & 4 & \\
\hline 15 & 1 & 15 & 3.8730 & & & & 4 & \\
\hline 18 & 1 & 18 & 4.2426 & & & 2 & & 4 \\
\hline 21 & 1 & 21 & 4.5826 & & & & & 4 \\
\hline 25 & 1 & 25 & 5 & & & & & 2 \\
\hline 30 & 1 & 30 & 5.4772 & & & & 4 & \\
\hline 42 & 1 & 42 & 6.4807 & & & & & 4 \\
\hline 50 & 1 & 50 & 7.0711 & & & & & 2 \\
\hline
\end{tabular}

lattices, it is known (Grimmer et al., 1974) that the multiplicity is odd, which together with the result $\Sigma_{I}=\Sigma_{P} / 2, \Sigma_{P}$ or $2 \Sigma_{P}$ determines $\Sigma_{I}$ uniquely in the two exceptional cases. The results are collected in Tables 8-10.

Notice that Table 8 is symmetric similar to Table 3: If $m$ is odd and $n$ an arbitrary integer, then $\mu=m, \nu=n$ and $\mu=2 n$, $v=m$ give rise to CSLs with the same values of $\omega$ for the same $\Sigma_{I}$ values. The row with $c^{2} / a^{2}=1$ corresponds to a lattice that metrically coincides with $c I$, the row with $c^{2} / a^{2}=2$ to a lattice that metrically coincides with $c F$. There exist two misorientations of these lattices with $\Sigma_{I}=3$ (and three with $\Sigma_{I}=5$ ) that are not equivalent with respect to the tetragonal holo- hedry, although they are equivalent with respect to the cubic holohedry.

Tables 9 and 10 give for each misorientation listed in Table 8 the quadruple representing the reduced rotation and the corresponding axes of $180^{\circ}$ rotations and twin mirror planes in the SST. The misorientations are arranged according to increasing values of $\Sigma_{I}$ and for a given value of $\Sigma_{I}$ according to increasing values of $c / a . i=2 \Sigma_{I} \cos \Theta$, where $\Theta$ is the misorientation angle corresponding according to equation (1) to the representative quadruple $(m, U, V, W)$.

Tables 9 and 10 allow us to read off all axes of $180^{\circ}$ rotations and all twin mirror planes of $t I$ twins by reticular merohedry, with $\Sigma_{I} \leq 5$, as well as the corresponding values of $c / a$. If the experimental value of the axial ratio slightly deviates from one of these values, then the corresponding laws describe twinning by reticular pseudomerohedry $(\delta>0)$.

\section{Application of Mallard's rule to tin}

Assume that we describe a twin law of a tetragonal crystal by one of the twin mirror planes $(h k l)$ with normal $[u v w]$ given in one of the Tables 4, 5, 9 or 10 . The corresponding axial ratio $c / a$ will be an approximation to the experimental value $(c / a)_{\text {exp. }}$. The formula for the obliquity $\delta$ given by Donnay \& Donnay (1959) simplifies for conventional tetragonal bases to

$$
\cos ^{2} \delta=\frac{(u h+v k+w l)^{2}}{\left(u h^{\prime}+v k^{\prime}+w l^{\prime}\right)\left(u^{\prime} h+v^{\prime} k+w^{\prime} l\right)} \frac{u^{\prime} h^{\prime}}{u h},
$$

where

$$
\left(h^{\prime} k^{\prime} l^{\prime}\right) \sim\left[u v\left(c^{2} / a^{2}\right)_{\exp } w\right], \quad\left[u^{\prime} v^{\prime} w^{\prime}\right] \sim\left[h k\left(a^{2} / c^{2}\right)_{\exp } l\right] .
$$

If $u^{\prime} h^{\prime} / u h=0 / 0$, it has to be replaced by $v^{\prime} k^{\prime} / v k$ or $w^{\prime} l^{\prime} / w l$ in equation (6).

Metallic tin ( $\beta$-Sn) has space group $I 4_{1} /$ amd and axial ratio $c / a$ increasing from 0.5456 at $306 \mathrm{~K}$ to 0.5477 at $485 \mathrm{~K}$ (Deshpande \& Sirdeshmukh, 1962). We shall compute the obliquity for $(c / a)_{\exp }=(0.3)^{1 / 2}=0.5477$.

I calculated $\delta$ for all misorientations of $t I$ with $\Sigma_{I} \leq 5$ and $0.4472 \leq c / a \leq 0.6547$. The calculations were carried out for twin mirror plane 1 in Tables 9 and 10 because $\delta$ has the same value for the twin mirror planes 1 and 2 . All but one of the solutions with $0.4714 \leq c / a \leq 0.5774$ yielded $\delta<6^{\circ}$, as shown in Table 11; the solutions with $c / a<0.4714$ or $>0.5774$ gave $\delta>6^{\circ}$.

According to Kirschstein (1971), the most common twin in $\beta$-Sn has twin mirror plane (301); less common are twins with mirror plane (101). Table 11 shows that both correspond to our solution with $c / a=0.5774, \Sigma_{I}=2$ and $\delta=2.6134^{\circ}$. Twins with mirror plane (101) have a second description satisfying Mallard's rule: $c / a=0.5, \Sigma_{I}=5$ and $\delta=4.1769^{\circ}$. The first description is more convincing: $c / a$ deviates less from the experimental value for $\beta$-Sn; $\Sigma_{I}$ and $\delta$ are smaller.

The program of Le Page (2002), available at http://ylp. icpet.nrc.ca/oblique/, carried out for $t I$ with $c / a=0.5477, \Sigma \leq$ 5 and $\delta \leq 6^{\circ}$, gives the solutions in our Table 1 with $\Sigma \leq 5$ and those in Table 11 with $\delta \leq 6^{\circ}$ (in two cases, $\Sigma_{I}=5, c / a=0.5$ and 
Table 9

Specific misorientations with multiplicity $\Sigma_{I} \leq 4$ for the $t I$ lattices.

\begin{tabular}{|c|c|c|c|c|c|c|c|c|c|c|}
\hline \multirow[b]{2}{*}{$\Sigma_{I}$} & \multirow[b]{2}{*}{$\mu$} & \multirow[b]{2}{*}{$v$} & \multirow[b]{2}{*}{$\omega$} & \multirow[b]{2}{*}{$c / a$} & \multicolumn{2}{|c|}{ Representative } & \multicolumn{2}{|c|}{ Twin mirror plane 1} & \multicolumn{2}{|c|}{ Twin mirror plane 2} \\
\hline & & & & & $(m, U, V, W)$ & $i$ & $(h k l)$ & {$\left[\begin{array}{lll}u & v & w\end{array}\right]$} & $(h k l)$ & {$\left[\begin{array}{lll}u & v & w\end{array}\right]$} \\
\hline \multirow[t]{2}{*}{1} & 1 & 1 & 2 & 1 & 1100 & 0 & 101 & 101 & & \\
\hline & 2 & 1 & 2 & 1.4142 & 1110 & 0 & 112 & 111 & & \\
\hline \multirow[t]{4}{*}{2} & 1 & 3 & 4 & 0.5774 & 3100 & 2 & 101 & 103 & 301 & 101 \\
\hline & 2 & 3 & 4 & 0.8165 & 3110 & 2 & 112 & 113 & 332 & 111 \\
\hline & 3 & 1 & 4 & 1.7321 & 1100 & 2 & 103 & 101 & 101 & 301 \\
\hline & 6 & 1 & 4 & 2.4495 & 1110 & 2 & 116 & 111 & 112 & 331 \\
\hline \multirow[t]{14}{*}{3} & 1 & 9 & 2 & 0.3333 & 3100 & 0 & 301 & 103 & & \\
\hline & 1 & 5 & 4 & 0.4472 & 5100 & 4 & 101 & 105 & 501 & 101 \\
\hline & 2 & 9 & 2 & 0.4714 & 3110 & 0 & 332 & 113 & & \\
\hline & 2 & 5 & 4 & 0.6325 & 5110 & 4 & 112 & 115 & 552 & 111 \\
\hline & 1 & 2 & 4 & 0.7071 & 2100 & 2 & 101 & 102 & 201 & 101 \\
\hline & 1 & 1 & 8 & 1 & 3111 & 3 & 211 & 211 & & \\
\hline & 1 & 1 & 4 & 1 & 2110 & 2 & 112 & 112 & 111 & 111 \\
\hline & 2 & 1 & 4 & 1.4142 & 1100 & 2 & 102 & 101 & 101 & 201 \\
\hline & 2 & 1 & 8 & 1.4142 & 3201 & 3 & 312 & 311 & & \\
\hline & 4 & 1 & 4 & 2 & 1110 & 2 & 114 & 111 & 112 & 221 \\
\hline & 5 & 1 & 4 & 2.2361 & 1100 & 4 & 105 & 101 & 101 & 501 \\
\hline & 9 & 1 & 2 & 3 & 1300 & 0 & 103 & 301 & & \\
\hline & 10 & 1 & 4 & 3.1623 & 1110 & 4 & 1110 & 111 & 112 & 551 \\
\hline & 18 & 1 & 2 & 4.2426 & 1330 & 0 & 116 & 331 & & \\
\hline \multirow[t]{20}{*}{4} & 1 & 15 & 4 & 0.2582 & 5100 & 2 & 301 & 105 & 501 & 103 \\
\hline & 2 & 15 & 4 & 0.3651 & 5110 & 2 & 332 & 115 & 552 & 113 \\
\hline & 1 & 7 & 4 & 0.3780 & 7100 & 6 & 101 & 107 & 701 & 101 \\
\hline & 1 & 4 & 2 & 0.5 & 2100 & 0 & 201 & 102 & & \\
\hline & 2 & 7 & 4 & 0.5345 & 7110 & 6 & 112 & 117 & 772 & 111 \\
\hline & 1 & 3 & 8 & 0.5774 & 3111 & 1 & 211 & 213 & & \\
\hline & 1 & 2 & 2 & 0.7071 & 2110 & 0 & 111 & 112 & & \\
\hline & 3 & 5 & 4 & 0.7746 & 5300 & 2 & 101 & 305 & 503 & 101 \\
\hline & 2 & 3 & 8 & 0.8165 & 3201 & 1 & 312 & 313 & & \\
\hline & 6 & 5 & 4 & 1.0954 & 5330 & 2 & 112 & 335 & 556 & 111 \\
\hline & 5 & 3 & 4 & 1.2910 & 1100 & 2 & 305 & 101 & 101 & 503 \\
\hline & 3 & 1 & 8 & 1.7321 & 3331 & 1 & 213 & 211 & & \\
\hline & 10 & 3 & 4 & 1.8257 & 1110 & 2 & 3310 & 111 & 112 & 553 \\
\hline & 4 & 1 & 2 & 2 & 1200 & 0 & 102 & 201 & & \\
\hline & 6 & 1 & 8 & 2.4495 & 3601 & 1 & 316 & 311 & & \\
\hline & 7 & 1 & 4 & 2.6458 & 1100 & 6 & 107 & 101 & 101 & 701 \\
\hline & 8 & 1 & 2 & 2.8284 & 1220 & 0 & 114 & 221 & & \\
\hline & 14 & 1 & 4 & 3.7417 & 1110 & 6 & 1114 & 111 & 112 & 771 \\
\hline & 15 & 1 & 4 & 3.8730 & 1300 & 2 & 105 & 301 & 103 & 501 \\
\hline & 30 & 1 & 4 & 5.4772 & 1330 & 2 & 1110 & 331 & 116 & 551 \\
\hline
\end{tabular}

Table 10

Specific misorientations with multiplicity $\Sigma_{I}=5$ for the $t I$ lattices.

\begin{tabular}{|c|c|c|c|c|c|c|c|c|c|}
\hline \multirow[b]{2}{*}{$\mu$} & \multirow[b]{2}{*}{$v$} & \multirow[b]{2}{*}{$\omega$} & \multirow[b]{2}{*}{$c / a$} & \multicolumn{2}{|c|}{ Representative } & \multicolumn{2}{|c|}{ Twin mirror plane 1} & \multicolumn{2}{|c|}{ Twin mirror plane 2} \\
\hline & & & & $(m, U, V, W)$ & $i$ & $(h k l)$ & {$[u v w]$} & $(h k l)$ & {$[u v w]$} \\
\hline 1 & 25 & 2 & 0.2 & 5100 & 0 & 501 & 105 & & \\
\hline 1 & 21 & 4 & 0.2182 & 7100 & 4 & 301 & 107 & 701 & 103 \\
\hline 2 & 25 & 2 & 0.2828 & 5110 & 0 & 552 & 115 & & \\
\hline 2 & 21 & 4 & 0.3086 & 7110 & 4 & 332 & 117 & 772 & 113 \\
\hline 1 & 9 & 4 & 0.3333 & 9100 & 8 & 101 & 109 & 901 & 101 \\
\hline 1 & 6 & 4 & 0.4082 & 3100 & 2 & 201 & 103 & 301 & 102 \\
\hline 1 & 5 & 8 & 0.4472 & 5210 & 0 & 211 & 215 & & \\
\hline 2 & 9 & 4 & 0.4714 & 9110 & 8 & 112 & 119 & 992 & 111 \\
\hline 1 & 4 & 4 & 0.5 & 4100 & 6 & 101 & 104 & 401 & 101 \\
\hline 1 & 3 & 4 & 0.5774 & 3110 & 2 & 111 & 113 & 332 & 112 \\
\hline 2 & 5 & 8 & 0.6325 & 5310 & 0 & 312 & 315 & & \\
\hline 3 & 7 & 4 & 0.6547 & 7300 & 4 & 101 & 307 & 703 & 101 \\
\hline 1 & 2 & 4 & 0.7071 & 4110 & 6 & 112 & 114 & 221 & 111 \\
\hline 2 & 3 & 4 & 0.8165 & 3200 & 2 & 101 & 203 & 302 & 101 \\
\hline 6 & 7 & 4 & 0.9258 & 7330 & 4 & 112 & 337 & 776 & 111 \\
\hline
\end{tabular}


Table 10 (continued)

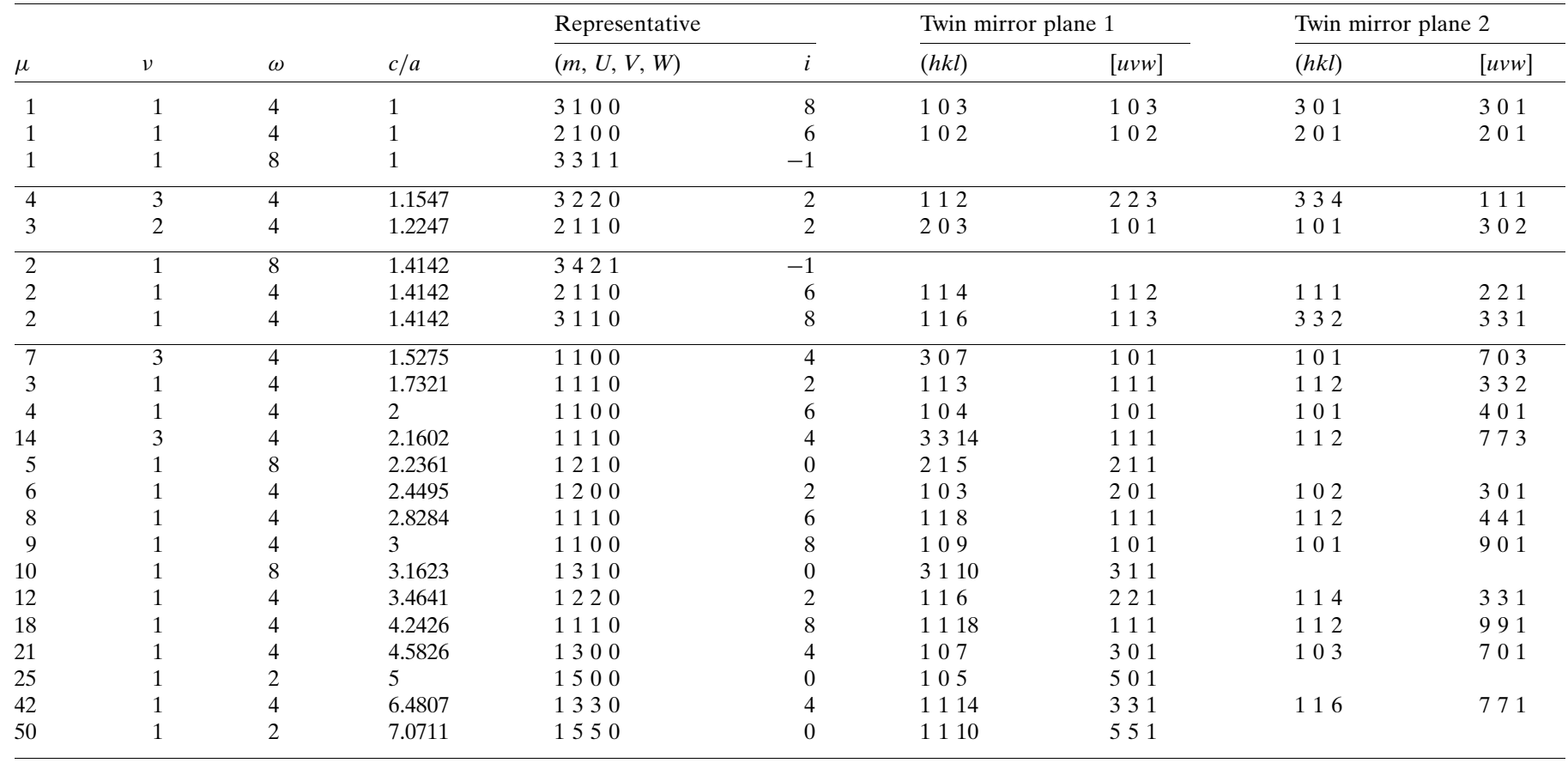

Table 11

Computation of the obliquity $\delta$ for $\beta$-Sn $\left[\left(c^{2} / a^{2}\right)_{\exp }=0.3\right]$ for the twin orientations with $\Sigma_{I} \leq 5$ of $t I$ lattices with $0.4714 \leq c / a \leq 0.5774$.

\begin{tabular}{llllllll}
\hline$c / a$ & $\Sigma_{I}$ & $h k l$ & $u v w$ & $h^{\prime} k^{\prime} l^{\prime}$ & $u^{\prime} v^{\prime} w^{\prime}$ & $\cos ^{2} \delta$ & $\delta^{\circ}$ \\
\hline 0.4714 & 3 & 332 & 113 & 10109 & 9920 & {$\left[12^{2} /(47 \times 94)\right] \times 30$} & 8.5653 \\
& 5 & 112 & 119 & 101027 & 3320 & {$\left[20^{2} /(263 \times 46)\right] \times 30$} & 5.1638 \\
& & 992 & 111 & & & & \\
\hline 0.5 & 4 & 201 & 102 & 503 & 305 & {$\left[4^{2} /(11 \times 11)\right] \times(15 / 2)$} & 5.2159 \\
& 5 & 101 & 104 & 506 & 3010 & {$\left[5^{2} /(29 \times 13)\right] \times 15$} & 4.1769 \\
& & 401 & 101 & & & & \\
\hline 0.5345 & 4 & 112 & 117 & 101021 & 3320 & {$\left[16^{2} /(167 \times 46)\right] \times 30$} & 0.9245 \\
& & 772 & 111 & & & & \\
\hline 0.5774 & 2 & 101 & 103 & 1009 & 3010 & {$\left[4^{2} /(37 \times 13)\right] \times 30$} & 2.6134 \\
& & 301 & 101 & & & & \\
& 4 & 211 & 213 & 20109 & 6310 & {$\left[8^{2} /(77 \times 25)\right] \times 30$} & 2.9213 \\
& 5 & 111 & 113 & 10109 & 3310 & {$\left[5^{2} /(47 \times 16)\right] \times 30$} & 2.9561 \\
& & 332 & 112 & & & & \\
\hline
\end{tabular}

solutions are collected in Table 12. They do not correspond to twins observed in $\beta$-Sn.

\section{Discussion of the results, conclusions}

If two lattices have primitive bases $\mathbf{e}_{i}$ and $\mathbf{f}_{i}, i=1,2,3$, satisfying $\mathbf{e}_{i} \cdot \mathbf{f}_{j}=k \delta_{i j}$, where $k$ is a constant of dimension length squared, then we shall call these two lattices pseudoreciprocal. Examples: the cubic lattices $c I$ and $c F$ are pseudoreciprocal; $c P$ is pseudoreciprocal to itself. The $t P$ lattice with $\mu=m, v=n$ is pseudoreciprocal to the $t P$ lattice with $\mu=n, v=$ $m$; the $t I$ lattice with $\mu=m, v=n$ ( $m$ odd) is pseudoreciprocal to the $t I$ lattice with

Table 12

Additional solutions satisfying Mallard's rule for $t I$ with $c / a=05477, \Sigma \leq$ 5 and $\delta \leq 6^{\circ}$ according to Le Page (2002).

\begin{tabular}{lllllll}
\hline$\Sigma_{I}$ & $h k l$ & $u v w$ & $h^{\prime} k^{\prime} l^{\prime}$ & $u^{\prime} v^{\prime} w^{\prime}$ & $\cos ^{2} \delta$ & $\delta^{\circ}$ \\
\hline 4 & 411 & 313 & 30109 & 12310 & {$\left[16^{2} /(127 \times 61)\right] \times 30$} & 5.3361 \\
4 & 118 & 001 & 001 & 3380 & {$\left[8^{2} /(1 \times 646)\right] \times 10$} & 5.5304 \\
4 & 433 & 113 & 10109 & 4310 & {$\left[16^{2} /(47 \times 55)\right] \times 10$} & 5.6437 \\
5 & 1110 & 001 & 001 & 33100 & {$\left[10^{2} /(1 \times 1006)\right] \times 10$} & 4.4293 \\
5 & 983 & 111 & 10103 & 9810 & {$\left[20^{2} /(23 \times 175)\right] \times 10$} & 4.5202 \\
\hline
\end{tabular}

0.4714 , one of our two solutions is missing). In addition, it gives those solutions that do not correspond to $[u v w] \perp(h k l)$ in a tetragonal lattice with an appropriate value of $c / a$. These $\mu=2 n, \nu=m$. We noticed that Tables 3, 4, 5, 8, 9 and 10 are symmetric with respect to the transition to pseudoreciprocal lattices. For a given misorientation (and hence a given value of $\Sigma$ ), the CSL (DSCL) for a tetragonal lattice is pseudoreciprocal to the DSCL (CSL) for its pseudoreciprocal lattice. This is a manifestation of the reciprocity relation proved by Grimmer (1974).

Formula (2) shows that $\Sigma_{P}$ remains invariant if we exchange $U \leftrightarrow V, m \leftrightarrow W$ or $\mu, m, W \leftrightarrow v, U, V$. Although we have not derived an analogous generally valid formula for $\Sigma_{I}$, it follows from the results of Singh \& King (1993) on equivalent quadruples that also $\Sigma_{I}$ must remain invariant if we exchange $U \leftrightarrow V$ or $m \leftrightarrow W$. On the other hand, $\Sigma_{I}$ is not invariant under $\mu, m, W \leftrightarrow v, U, V$. 
Even without a generally valid formula for computing $\Sigma_{I}$, we have been able to derive a convenient lower limit for the multiplicity $\Sigma_{I}$ of specific coincidence misorientations as a function of $\mu$ and $\nu$, which makes it possible to determine all misorientations with multiplicity up to a specified limit.

The lower limits on $\Sigma_{P}$ and $\Sigma_{I}$ have been used to list the possible twin laws with $\Sigma \leq 5$ of tetragonal crystals by reticular merohedry, which implies that the obliquity $\delta=0$. For most twin laws, this is possible only for a certain axial ratio $(c / a)_{0}$, the square of which is rational. For a crystal with axial ratio close to $(c / a)_{0}$, the twin law under consideration leads to $\delta>0$, i.e. to reticular pseudomerohedry, as discussed for $\beta$-Sn.

Not all twin laws by reticular pseudomerohedry with $\Sigma \leq 5$ and $\delta \leq 6^{\circ}$ are related to twin laws by reticular merohedry with $\Sigma \leq 5$, as shown in Table 12 . However, the results for tin and unpublished results for cubic crystals and quartz suggest that most experimentally found twin laws by reticular pseudomerohedry of cubic, tetragonal, hexagonal and trigonal crystals are related to reticular merohedry. This issue needs further investigation.
Stimulating comments by Elke Koch and Karsten Kunze on an earlier version of this paper are gratefully acknowledged.

\section{References}

Bollmann, W. (1967). Philos. Mag. 16, 383-399.

Deshpande, V. T. \& Sirdeshmukh, D. B. (1962). Acta Cryst. 15, 294-295.

Donnay, J. D. H. \& Donnay, G. (1959). International Tables for X-ray Crystallography, Vol. II, ch. 3. Birmingham: Kynoch Press.

Fischmeister, H. F. (1985). J. Phys. Paris, 46, C4-3-C4-23.

Friedel, G. (1926). Leçons de Cristallographie. Reprinted 1964. Paris: Blanchard.

Grimmer, H. (1974). Scr. Metall. 8, 1221-1224.

Grimmer, H. (1980). Acta Cryst. A36, 382-389.

Grimmer, H. (1989). Scr. Metall. 23, 1407-1412.

Grimmer, H. (1993). Mater. Sci. Forum, 126-128, 269-272.

Grimmer, H., Bollmann, W. \& Warrington, D. H. (1974). Acta Cryst. A30, 197-207.

Kirschstein, G. (1971). Gmelins Handbuch der Anorganischen Chemie, Vol. Zinn, Part B. Weinheim: Verlag Chemie.

Le Page, Y. (2002). J. Appl. Cryst. 35, 175-181.

Mallard, E. (1885). Bull. Soc. Minéral. Fr. 8, 452-469.

Singh, A. \& King, A. (1993). Acta Cryst. B49, 266-272. 